\title{
Putting Data Linkage into FIRST Gear: Lessons from Firefighter Injury Research
}

\author{
Jennifer A. Taylor ${ }^{\star 1}$, Michael T. Le Vasseur ${ }^{1}$, Shannon A. Widman ${ }^{1}$, Priya Sankar² and \\ Henry J. Costo ${ }^{3}$
}

${ }^{1}$ Department of Environmental \& Occupational Health, Drexel University Schoof of Public Health, Philadelphia, PA, USA; ${ }^{2}$ Office of the General Counsel, Drexel University, Philadelphia, PA, USA; ${ }^{3}$ Fire Department, City of Philadelphia, Philadelphia, PA, USA

\section{Objective}

The purpose of this panel is to describe the process of using data to develop firefighter nonfatal injury surveillance systems in the city of Philadelphia and the state of Florida through the linkage of data from workers' compensation, inpatient and emergency department hospitalizations, human resources, and continuing education/training registries.

\section{Introduction}

This work builds on a successful demonstration project and expands its data linkage capacity to new community partners. Presently, a national non-fatal injury reporting system does not exist for the Fire Service. In order to tell the story of all injuries within a fire department, state, or on a national scale, we must utilize data that are available from multiple sources that do not naturally talk to each other. In this panel, we will describe the purpose of the project, its goals, and the success of its model to public health surveillance.

\section{Description}

We will engage the audience through a panel presentation comprised of members of our team who were critical to each step in the process, from stakeholder engagement to data acquisition and linkage. We will describe, in a stepwise process, how we effectuated data agreements, educated and heard from stakeholders, and ultimately analyzed the resulting data to uncover conclusions regarding injuries in the Fire Service. Panelists include a legal expert, a statistician, an industry partner, and project staff. The panel will begin with the discussion of the various stakeholders necessary (including leadership from national organizations) to garner agreements on the importance and benefits of data linkage. We will discuss the legal process and implications of establishing data sharing agreements and contractual relationships, especially around the access to personally identifiable health information (PHI). We will explore issues such as access to personal identifiers, and how working with stakeholders to examine policy facilitators and barriers to accessing data, in order to perform data linkage between data sets that do not regularly talk to each other, is critical for driving the process forward. We will also discuss the utility of the resultant master database to the community partners, and how we elicit "givebacks" to the data stewards so that they understand the utility and relevance of the databases created. We will then move on to show how the PHI data linkage was conducted using direct and probabilistic data linkage methods. We will describe our computing environment, especially as it pertains to data security and integrity. We will conclude with a description of the analyses we were able to undertake because we successfully linked data sets that had not previously talked to one another. We will also integrate one of our community stakeholders to share his perspective on the utility of the resultant data for his prevention priorities for the road ahead.

\section{Audience Engagement}

Methods for cultivating relationships with target communities and stakeholders will be discussed by project staff members (Jennifer Taylor/Shannon Widman).

The legal process, hurdles, and implications, regarding effectuating data sharing agreements with target sites, will be presented by a legal expert (Priya Sankar).

Data linkage, requirements and methods, will be explored by an experienced statistician (Michael LeVasseur).

The utility and usefulness of the final product resulting from the data linkage will be presented by one of our project's stakeholders, a Fire Service community member (Henry Costo).

\section{Keywords}

data linkage; surveillance; fire service

\author{
*Jennifer A. Taylor \\ E-mail: jat65@drexel.edu
}

